\title{
Transient Non-Autoimmune Hyperthyroidism of Early Pregnancy
}

\author{
Alexander M. Goldman and Jorge H. Mestman \\ Department of Medicine, Keck School of Medicine, University of Southern California, Los Angeles, CA 90033, USA \\ Correspondence should be addressed to Jorge H. Mestman, mestman@usc.edu
}

Received 22 March 2011; Revised 4 May 2011; Accepted 4 May 2011

Academic Editor: Roberto Negro

Copyright ( $) 2011$ A. M. Goldman and J. H. Mestman. This is an open access article distributed under the Creative Commons Attribution License, which permits unrestricted use, distribution, and reproduction in any medium, provided the original work is properly cited.

It is characterized by chemical and sometimes clinical hyperthyroidism, without evidence of thyroid autoimmunity that resolves spontaneously by 16 weeks gestation without significant obstetrical complications.

\section{Introduction}

Common Presentation. 26 y/o women, G1, P0, at 8 weeks gestation presents to the physician with a 3 weeks history of nausea and vomiting, weight loss of $2 \mathrm{~kg}$., unable to tolerate solid foods, mild hands tremor, and palpitations with a pulse rate of 104 beats per minute. Serum TSH $0.02 \mathrm{mIU} / \mathrm{L}$ and FT4 $2.8 \mathrm{ng} / \mathrm{cl}(0.9-1.8)$.

For the last few years, thyroid function tests have been frequently requested in early pregnancy because of physicians' awareness of potential obstetrical complications, both maternal and fetal, with an additional concern about the neuropsychological well-being of children born of mothers with thyroid dysfunction. Although universal screening is not recommended by some obstetric and endocrine societies, an aggressive detection program based on medical history and physical examination (presence of goiter) is encouraged. The interpretation of thyroid function tests in early pregnancy needs to be assessed in the context of the physiopathological changes taking place. Perhaps the most striking difference in normal pregnancies, from nonpregnancy thyroid test values, is the significant lowering of serum TSH, due to TSH-like activity of human chorionic gonadotropin (hCG). Serum TSH in the first trimester, particularly between 7 and 12 weeks gestation, fall to a nadir and present a mirror image with peak hCG values [1-3]. In a recent review, the lower normal TSH limit of approximately $0.03-0.08 \mathrm{mIU} / \mathrm{L}$ in the first trimester of pregnancy was derived from several studies using trimester-specific reference ranges [4]. Therefore, a "low serum TSH" in the first trimester of gestation should be considered "physiologic" in the presence of normal serum-free thyroxine (FT4) value. The exception could be a woman with T3 hyperthyroidism due to an autonomous or "hot" thyroid nodule. Thyroid tests in the hyperthyroid range may be seen in the first trimester of pregnancy in women without previous or present history of Graves' disease; they present to the consult with a clinical spectrum from no symptomatology, to morning sickness, to different degrees of vomiting sometimes severe as in the syndrome of hyperemesis gravidarum. Thyroid tests could be quite abnormal, presenting a challenge to the physician in the differential diagnosis and management of such individuals.

In this paper, we will discuss the following:

(i) definition,

(ii) causes or etiologies,

(iii) clinical and laboratory diagnosis,

(iv) management.

An emphasis will be placed on early series of women affected, on the difficulty in the differential diagnosis from Graves' hyperthyroidism and its management, and on the evolution of the role of hCG in its pathogenesis through the years.

We decided to use the term "transient nonimmune hyper-thyroidism of early pregnancy" because of its multiple 
TABLE 1: Transient nonautoimmune hyperthyroidism in early pregnancy.

\begin{tabular}{ll}
\hline (i) & Normal pregnancy \\
(ii) & Mild nausea and vomiting \\
(iii) & Hyperemesis gravidarum (transient hyperthyroidism of \\
(iv) & Twin or multiple pregnancies \\
(v) & Mutation in the TSH Receptor \\
(vi) & Hyperplacentosis \\
(vii) & Hyperreactio luteinalis \\
(viii) & Hydatidiform mole \\
(ix) & Choriocarcinoma \\
\hline
\end{tabular}

etiologies although the etiology related to hyperemesis gravidarum is the most common. Different names have been suggested for this clinical entity. In 1992, Goodwin et al. used the term "transient thyrotoxicosis of hyperemesis gravidarum" [5, 6]; "gestational thyrotoxicosis" was proposed in 1993 as a new clinical entity by Kimura et al. [7]. Other nomenclatures were and are still employed when describing the syndrome, such as gestational hyperthyroidism (GH) and gestational transient thyrotoxicosis (GTT). In most of these reports, the common findings are vomiting of different intensity and thyroid tests in the hyperthyroid range without evidence of thyroid autoimmunity.

\section{Definition}

Hyperthyroidism diagnosed for the first time in early pregnancy, transient, without evidence of thyroid autoimmunity, lack of physical findings consisting with Graves' disease, resolving spontaneously by the end of the first or early second trimester of pregnancy.

\section{Etiology}

In the first trimester of pregnancy, several situations may present in which thyroid tests are consistent with hyperthyroidism in the absence of either autoimmune thyroid disease or an autonomous or functioning thyroid adenoma (Table 1).

3.1. Morning Sickness (Nausea and Vomiting) in Early Pregnancy. Mori et al. [8] reported in 1988 the relationship between morning sickness and thyroid function in pregnancies not affected by autoimmune thyroid disease. They studied 132 women in early pregnancy and compared them to 20 nonpregnant controls. Pregnant women were grouped in (a) no symptoms, (b) nausea only, and (c) nausea and vomiting. Serum-free T4 (FT4), hCG and serum thyrotropin stimulating hormone (TSH) with assay sensitivity of $0.1 \mu \mathrm{U} / \mathrm{m}$ were measured. The authors concluded that the increased serum concentration of free T4 and hCG and decreased serum TSH correlated with the severity of morning sickness. Unfortunately, the authors did not report the percent of patients in each group with values outside the normal range.
Transient hyperthyroidism of hyperemesis gravidarum (THHG)

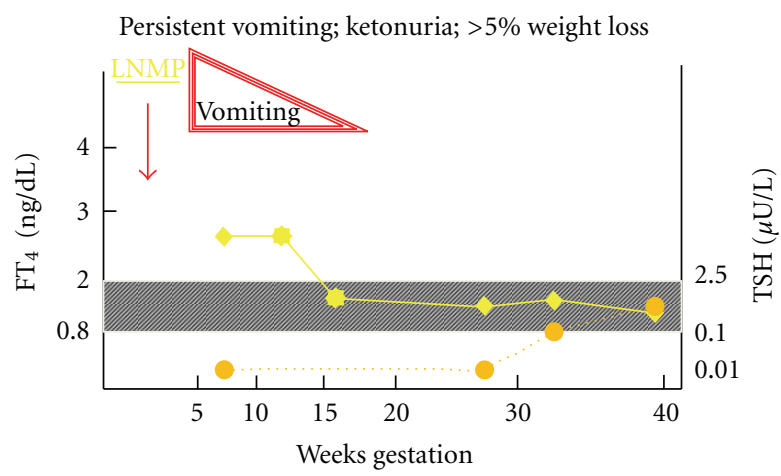

FIGURE 1: A representative example of transient hyperthyroidism of hyperemesis gravidarum. By week 6, vomiting begins and becomes severe by week 10 . Serum-free thyroxine (T4) index is elevated and thyrotropin is suppressed. By weeks 16 to 18 , vomiting subsides with marked improvement of the free T4 index value. During this period, the patient loses $3.6 \mathrm{~kg}$. By week 18, the serum free T4 index returns to normal, but the serum thyrotropin remains suppressed until week 26. Patient regains and gains weight with a term delivery of a healthy infant. The gray band indicates reference range. LNMP: last normal menstrual period. Patil-Sisodia and Mestman [12].

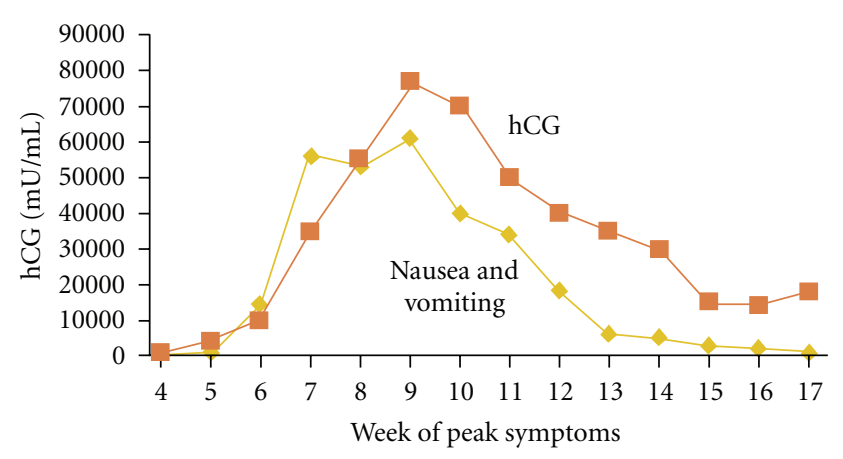

Figure 2: Relation between peak nausea and vomiting symptoms and human chorionic gonadotropin (hCG) levels. Niebyl [10].

3.2. Transient Hyperthyroidism of Hyperemesis Gravidarum (THHG). Hyperemesis gravidarum (HG) is reported to occur in $0.3 \%$ to $1.0 \%$ of pregnancies; it is defined as persistent nausea and vomiting resulting in greater than $5 \%$ weight loss, ketonuria, dehydration, and electrolytes (hypokalemia, metabolic alkalosis, hyponatremia, and hypochloremia), and liver abnormalities in severe cases $[9,10]$. The onset of nausea is within 4 weeks after the last menstrual period, with worsening by 9 weeks gestation, resolution by the end of the first trimester in $60 \%$ of cases, and complete resolution by 20 weeks in the vast majority of women (Figure 1). Decreased risk of miscarriage has been reported in women with nausea and vomiting [11]. The clinical course of nausea and vomiting during pregnancy correlated closely with the level of hCG (Figure 2) [10]. The cause of nausea and vomiting is unclear although high levels of estrogen and/or Vitamin B deficiency have been implicated. 


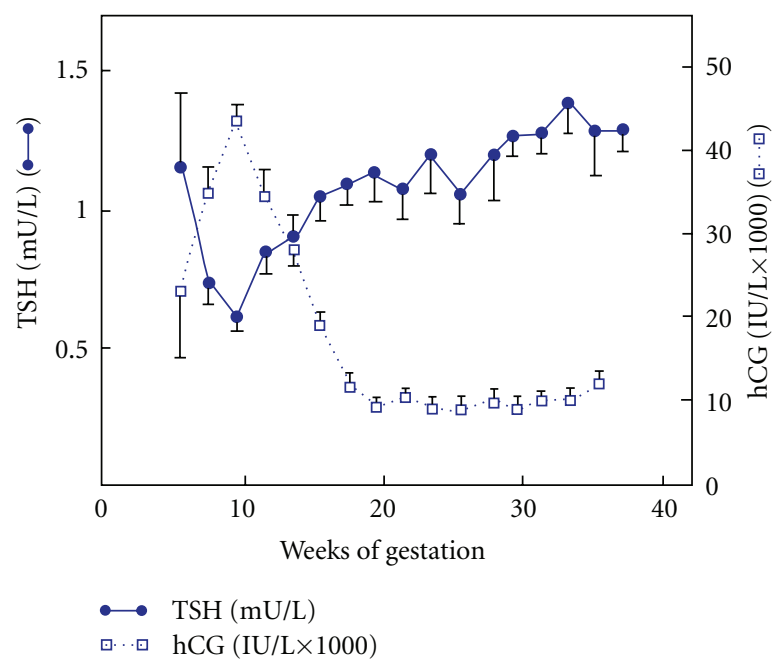

Figure 3: Serum hCG and TSH as a function of gestational age Glinoer et al. [1].

Ethnic variation in the incidence of HG has been suggested by several studies. For example, a birth registry in Norway from 1967 to 2005, revealed an overall prevalence of $0.9 \%$; however, when broken down by ethnicity, it affected $2.2 \%$ of Pakistani women; $1.9 \%$ of Turkish women, and $0.5 \%$ of Norwegian women [14]; a California study suggested a lower incidence in Caucasian and Latina women as compared to nonwhites or non-Hispanics [15]. A familial aggregation was suggested by Zhang et al. [16] in a study in which women were recruited through advertising on the Hyperemesis Education and Research Foundation Web Site. In their study, sisters of women with HG have a significantly increased risk of having $\mathrm{HG}$ themselves (OR, 17.3; $P=$ 0.005); mothers were also more affected than controls $33 \%$ versus $7.7 \%(P<0.001)$. The authors suggested that the study provides strong evidence for a genetic component of HG. A report from the same group, described three women with a history of HG, developing severe nausea and vomiting during ovarian stimulation for gestational surrogacy [17].

In a high percent of women affected by HG, (30 to 73\%), abnormal thyroid tests consistent with hyperthyroidism are detected; indeed, HG is the most common cause of THHG. The incidence of hyperthyroidism depends on the severity of symptoms, ethnic background, perhaps dietary iodine intake, interpretation of thyroid tests, and other unknown factors. The diagnosis of THHG is based on the presence of clinical and physical clues: the most common physical findings are tachycardia which is the result of dehydration and improved after fluid and electrolytes replacement, with average pulse rate of about $100 \mathrm{bpm}$ after hydration in the vast majority of patients; fine tremor of fingers may be present as well as mild proximal weakness. In cases with significant elevations of thyroid hormones, particularly in those with high serum T3 values, other symptoms such as shortness of breath, heat intolerance, and palpitations may be present. Characteristically, no goiter or Graves' ophthalmopathy are detected. There is an elevation in serum FT4 or free thyroxine index (FT4I), a suppressed or undetectable serum TSH and no markers of thyroid autoimmunity. Serum TT3 is slightly elevated in less than $20 \%$ of affected women. Until a few years ago, a suppressed serum TSH was considered diagnostic of THHG in spite of a normal serum FT4. With the improvement in the sensitivity of immunoassay techniques, the serum TSH in normal first trimester of pregnancy could be as low as $0.03-0.08 \mathrm{mIU} / \mathrm{L}$ secondary to the thyrotropic activity of hCG; indeed, there is an inverse relationship between serum levels of hCG and $\mathrm{TSH}$, and serum TSH exhibit a mirror image to the hCG peak (Figure 3).

The development of hyperthyroidism is most likely due to the thyrotropic action of hCG, its thyroid-stimulating activity as shown in bioassays in mice, rats, chicks, and men [18]. hCG stimulates iodine uptake and adenylate cyclase, and DNA synthesis in cultured rat thyroid cells. These studies demonstrated unequivocally that hCG activates the TSH receptor and is a weak thyrotropin. The thyrotropic activity of hCG is influenced by the metabolism of the hCG molecule, particularly by the number and structure of the oligosaccharide side chains. Deglycosylation and/or desialylation of hCG enhance its thyrotropic potency in rat (FRTL-5) thyroid cells. As discussed by Yoshimura et al. [19] the thyrotropic activity of hCG is regulated by two factors: the amount of desialylated hCG produced from trophoblast cells and its plasma half-life. hCG molecules that are less sialylated activate the TSH receptor to a greater extent, as has been reported in patients with the syndrome of THHG [20]. In bioassays, hCG is only about $1 / 10^{4}$ as potent as human TSH during normal pregnancy; it is likely that the thyrotropic activity of hCG during this peak secretion overrides the normal operation of the hypothalamic-pituitary feedback system [20].

Kauppila et al. [21], in 1979, reported on women with hyperemesis gravidarum, and how their serum hCG concentrations compared to those in a group of normal pregnant women. The 42 women with hyperemesis gravidarum were compared to 115 women with normal pregnancies during various periods in the first 20 weeks of pregnancy. In women with hyperemesis gravidarum, compared to normal pregnant women of matched gestational age, their mean HCG concentrations were higher at 7-8 weeks, 9-11 weeks, and 1214 weeks, but there was no difference found between the mean serums concentrations when women were tested at 1520 weeks. The authors suggested a causal relation between a high serum HCG concentration and HG.

Kennedy et al. [22] and Kimura [7] reported on patients with HG whose serum contained an activity, which stimulated cyclic AMP (cAMP) accumulation in cultured human thyroid cells and cultured FRTL- 5 cells, but the authors were unable to identify the thyroid stimulator. The thyroid stimulating properties of the sera were immediately abolished by addition of anti-hCG antibody [7]. Several investigators suggested that Asialo-hCG with higher thyrotropic bioactivity was the cause of the syndrome, explaining the poor correlation between HCG serum concentrations and clinical symptoms reported in some studies $[9,23,24]$. Jordan et al. [25] suggested that more acidic isoforms of hCG with longer 
half-life might result in more robust thyrotrophic effects in women with hyperemesis.

The first clinical description of an association between HG and hyperthyroidism was reported in 1978 by Bruun and Kristofferson [26]. The authors first considered the possibility of a related cause for the symptoms of hydatidiform mole and hyperemesis gravidarum and studied thyroid function tests. They found that in both conditions, there is an accompanying increase in serum hCG concentrations. Their findings that thyroid function abnormalities varied with hCG concentrations when compared to normal pregnancies, and opened the door to further clinical studies of hCG as a cause for the thyrotoxicosis that accompanies hyperemesis. They studied 35 women with HG, 14 with hydatidiform mole, and 57 normal pregnancies, as controls. They reported high values of protein binding iodine (PBI) in most patients with hydatidiform mole and in almost one third of HG women; in addition, they noticed lower serum cholesterol levels. They concluded "that there may be a common cause of the thyroid stimulation in patients with mole and hyperemesis." Soon thereafter, several reports of isolated or few cases of hyperthyroidism and HG were reported, showing the diagnostic challenges presented to physicians in the differential diagnosis from Graves' hyperthyroidism and the difficulties in management. In 1980, Valentine and Jones [27] demonstrated a case of a patient that began to establish the transient, self-limiting nature of hyperemesis gravidarum as well as the difficulty in establishing a definitive differential diagnosis from Graves' hyperthyroidism. The patient was treated with intravenous fluids to correct her severe dehydration. After developing pyrexia and tachycardia, her thyroid parameters were tested. She had an elevated FT4 index (FT4I) and long-acting thyroid stimulator (LATS), later recognized to be thyroid stimulating immunoglobulins (TSI), was negative; she was diagnosed with thyrotoxic crisis at 13 weeks' gestation and treated with carbimazole (CZ), propranolol, and Lugol's solution to normalize her thyroid function. The authors commented on several reports in the literature of patients with vomiting as the "cardinal symptom" of thyrotoxicosis [28]. Dozeman et al. [29] reported one patient during two pregnancies with severe HG (hypokalemia and abnormal liver tests), with similar clinical outcome, treated with ATD during the first but not during the second pregnancy, with normalization of thyroid tests at about the same gestational age; the authors diagnosis in the first pregnancy was Graves' hyperthyroidism and silent thyroiditis in the second pregnancy. Another case of two pregnancies in a patient with negative antibodies was reported with the same diagnostic dilemma as in previous cases [30].

By 1982, the first series specifically seeking to show the prevalence of hyperthyroxinemia accompanying HG (for purposes of this study, defined as irresistible vomiting for several weeks accompanied by a reduction in weight) was examined [31], showing that in $73 \%$ of 33 consecutive pregnancies complicated by hyperemesis gravidarum patients had some degree of hyperthyroxinemia, as determined by FT4I elevation. Important observations were pointed out by the authors: (a) serum FT3 was elevated in only 4 of 11 patients in whom FT3 was measured, (b) a blunted response to TRH test was seen in 5 hyperthyroxinemic women tested, (c) goiter, exophthalmos, or previous history of hyperthyroidism was absent in all patients, (d) a lower birth weight was observed in children born to hyperthyroxinemic mothers (3166 $\pm 501 \mathrm{gm}$, mean $\pm \mathrm{SD} ; n=19)$ versus pregnancies not complicated by HG $(3420 \pm 501 \mathrm{gm} ; n=828)(P<$ 0.01 ), findings not related to the use of ATD therapy, and (e) six mothers were treated with methimazole until normalization of thyroid tests, mean of 17 days with a range of 8 to 33 days, as compared to 19 days, range 6 to 46 days, in those women not receiving ATD therapy.

Juras et al. [32] reported an increased serum reverse Triiodothyronine (rT3) in women with HG, as compared to control women, suggesting an enhanced peripheral conversion of T4 to rT3, later confirmed by other studies $[33,34]$. Elevation in serum nonesterified fatty acids (NEFA) and rT3 were postulated as protection against further weight loss and lipolysis [33].

A study of 10 HG patients in 1984 showed the great variability in thyroid parameters that accompany hyperemesis gravidarum [34]. Approximately half of the patients had both abnormally elevated serum FT4I and elevated reverse T3 (rT3) levels, or blunted TRH response, representing a THHG prevalence of approximately $50 \%$ among hyperemetic women. Peak TSH response to the thyrotropin releasing hormone (TRH) test inversely correlated to serum hCG concentrations, while no correlation was found between rT3 levels and TSH response. The lack of elevated FT3I in the patients could also be a result of the same T4 to T3 conversion blockade responsible for elevated rT3 levels due to reduced caloric intake. Though ATD therapy was not pursued, all women delivered normal term infants.

A series of 25 HG patients [35] in 1986 included ten patients, $40 \%$ of them, developing THHG, defined as elevated FT4 concentrations, all of whom also had blunted responses to TRH stimulation test and rT3 concentrations elevated above the reference range. A further six patients who had FT4 concentrations within the reference range also had blunted TSH response to TRH, including four with responses as blunted as in the thyrotoxicosis patients. No ATD treatment was given, and all thyrotoxic patients' thyroid parameters resolved spontaneously.

In 1987, a THHG prevalence of $43.6 \%$ was reported in a series of 39 patients with HG [36]. There was also evidence of abnormal liver function in some patients. There were similar pregnancy outcomes between the thyrotoxic and euthyroid HG patients, with a slightly elevated incidence of iron deficiency anemia among the thyrotoxic group. Two patients had persistent hyperthyroidism and were treated with ATD therapy.

In 1992, the largest series of HG women with hyperthyroidism was published [5]. Of the $67 \mathrm{HG}$ patients $66 \%$ of them had biochemical hyperthyroidism, defined as either FT4I higher than the upper range of normal $(N=39)$, or a TSH less than $0.4 \mathrm{mU} / \mathrm{L}(N=60)$, Twenty of the patients had undetectable TSH levels, $<0.04 \mathrm{mU} / \mathrm{L}$. Forty-two percent of women had abnormal liver function tests and 6\% of them had an elevation in serum amylase titers. Abnormal 


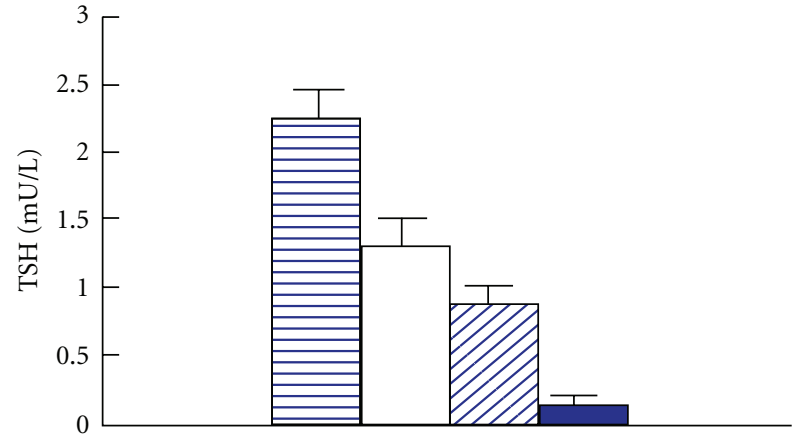

(a)

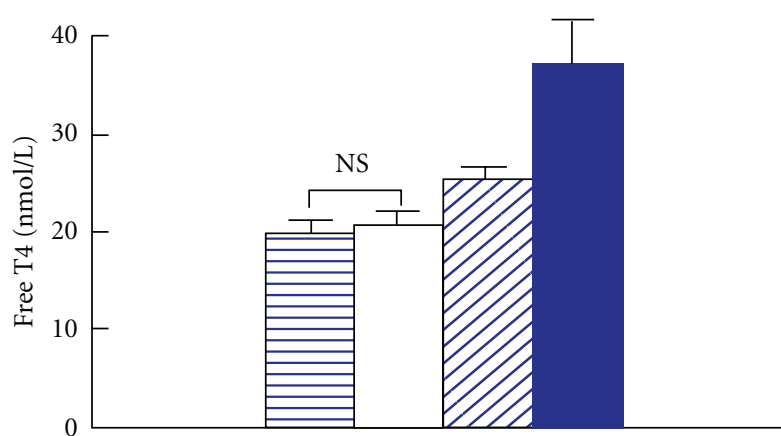

(b)

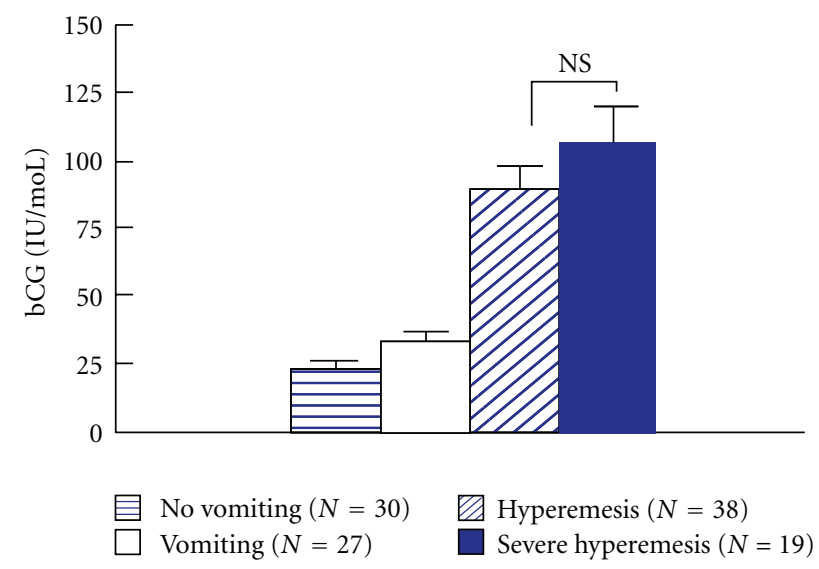

(c)

FIGURE 4: Relation between the severity of vomiting and serum concentrations of TSH, free T4, and hCG (mean \pm SE). Hormone concentrations differed significantly between each group of patients except as indicated by NS Goodwin et al. [13].

electrolytes, hyponatremia, hypokalemia, and elevated bicarbonate levels were found in $28 \%$ of cases, mostly related to the degree of dehydration due to vomiting. FT3I elevation was detected in only 6 women related to severity of the reported vomiting. No patient received ATD therapy, and all had spontaneous resolution of vomiting in no more than 18 weeks gestation. In all cases, biochemical hyperthyroidism normalized by the time vomiting ceased; however, in seven cases, vomiting continued for two to fourteen weeks after normalization of thyroid function. From those same 67 patients, a series of $57 \mathrm{HG}$ patients were selected and compared to 57 controls matched for gestational age and parity [13]. The authors divided the 57 women in four groups: no vomiting ( $n=30)$, mild vomiting $(n=27)$, hyperemesis gravidarum ( $n=38)$, and severe hyperemesis gravidarum $(n=19)$. Mean serum concentrations of hCG, FT4, estradiol, and prolactin (PRL) were all elevated in the HG groups. In Figure 4, the relationship between the severities of vomiting as indicated by clinical and biochemical parameters and the degree of thyroid dysfunction is shown. More severe vomiting was associated with a greater degree of thyroid stimulation and greater concentration of hCG. Serum from patients with HG was bioassayed for thyrotropic activity in thyroid cell culture, demonstrating a significant correlation between the serum concentration of hCG and the iodine uptake (Figure 5).

In 1993, Kimura et al. [7] studied 51 pregnant women divided into three groups based on the severity of their emesis symptoms, similar to the Goodwin et al. [13] protocol, their results confirmed previous findings of high serum FT4 and suppressed TSH, and high serum FT3 values in a few women. Interestingly, most of the patients in the emesis and hyperemesis group had undetectable serum TSH $(<0.01 \mathrm{mIU} / \mathrm{L})$. One difference in their study was that levels of hCG were not statistically different in the 3 groups. Two of the women in the hyperemesis group, who had both the highest FT4 concentration and thyroid-stimulating activity to hCG ratio, had clinical symptoms of thyrotoxicosis, which resolved as thyroid-stimulating activity and FT4 levels normalized. The authors suggested that a molecular variant of hCG with higher thyrotrophic activity was the cause of this syndrome.

Increased risk of THHG by ethnicity was evaluated by several investigators; among 294 South Asian women, from Pakistan, Bangladesh, or India, suppressed TSH concentrations $<0.35 \mathrm{mIU} / \mathrm{L}$ were found in $15.7 \%$, compared to $4.8 \%$ of 292 age and parity-matched European women, suggesting that South Asian women may be at higher risk for gestational 


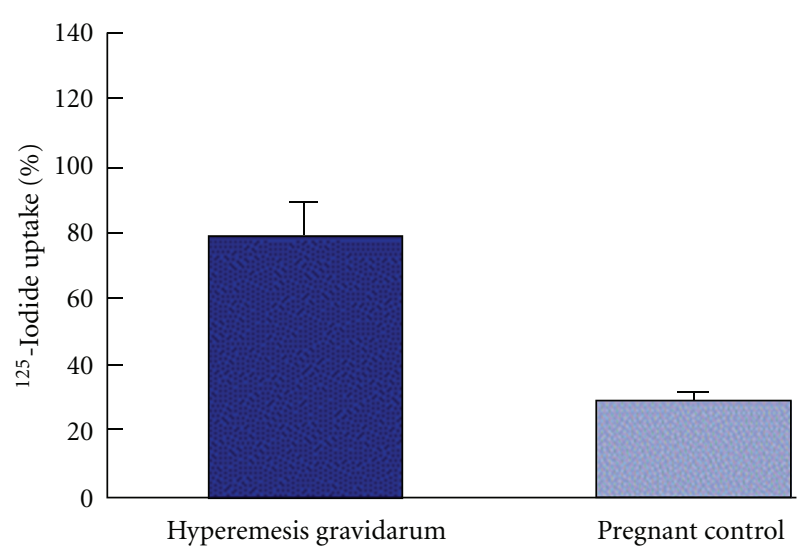

(a)

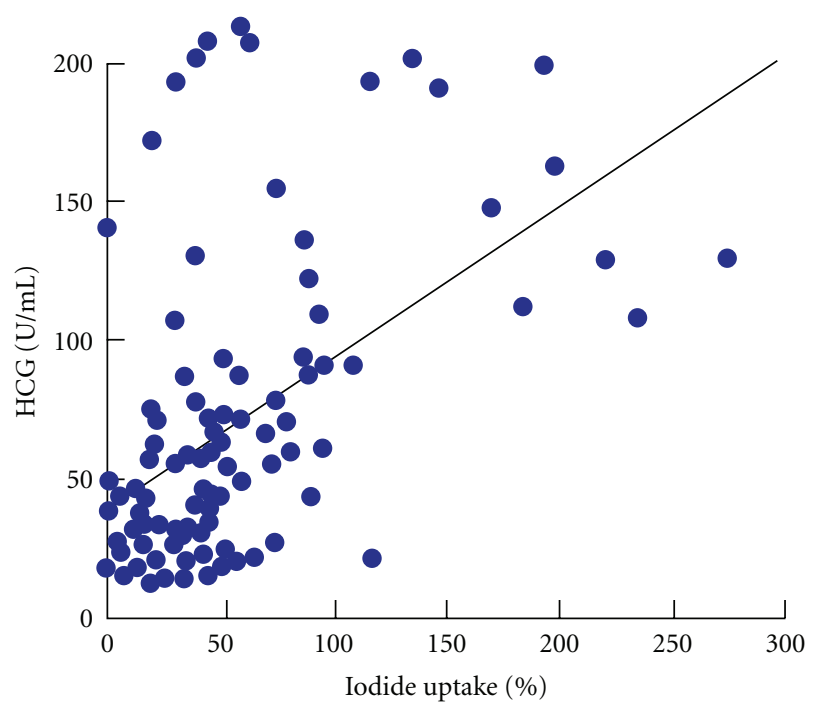

(b)

FIgURE 5: (a) Serum thyrotropic activity (measured as iodide uptake in cultured rat cells) in hyperemesis patients and pregnant controls, mean \pm SE, $P<0.001$ ) (b) correlation of serum hCG versus serum thyrotropic activity in hyperemesis and pregnant controls, $r=0.50$, $P<0.001$ Goodwin et al. [13].

thyrotoxicosis [37]. However, with the use of more sensitive assay for the determination of serum TSH, some of the patients with serum TSH $<0.35$, considered "suppressed" by the authors could have had normal values. The higher prevalence by ethnicity was confirmed by other studies [38].

A review of previous series and a case report was undertaken in 2000, confirming that THHG generally resolves by 18 weeks' gestation without ATD treatment and without complicating the outcome of the pregnancy. The author suggested four clinical criteria for the diagnosis of HGGT: (1) abnormal thyroid function tests developing in the context of HG, (2) no evidence of prepregnancy hyperthyroidism, (3) absence of physical examination findings consistent with hyperthyroidism, and (4) negative thyroid autoantibodies titers [39].

A female predominance among the offspring of mothers with HG was reported by Deruelle et al. [40]. Of the 33 patients admitted with HG, 23 of them (66.7\%) had THHG. The authors speculated about the involvement of hyperthyroidism and fetal sex in the pathogenesis of hyperemesis gravidarum.

Panesar et al. [41] attempted to determine the causal relationship between hCG and thyroid hormones in causing HG in Chinese women. A group of $58 \mathrm{HG}$ women were compared to a gestational-age-matched group of 58 pregnant controls, and it was found that maternal age and all hormones were significantly different between the hyperemetic and control groups. Only FT4, sensitive TSH (sTSH), and maternal age were found to be significant independent variables.

In 2002, a study was done of 53 Asian women, from Singapore, who presented with $\mathrm{HG}$ and was later found to have accompanying hyperthyroidism [42]. Five had Graves' disease, and nine were excluded because of incomplete followup. In the remaining 39 women, none had clinically hyperthyroidism; there was a high incidence of abnormal electrolytes $(60 \%)$ and abnormal liver function tests $(56 \%)$. Without ATD therapy, FT4 levels normalized by 15 weeks' gestation, and TSH levels normalized by 19 weeks. There were no preterm deliveries, and a median birth weight of $2970 \mathrm{~g}$, with a male-to-female ratio of $3: 1$. Birth weight was lower in mothers experiencing a weight loss of $>5 \%$ of their prepregnancy weight, as compared to women who did not, however the difference was not significant $(P=0.093)$.

A case was reported in 1996 of a single patient with HG in three successful pregnancies, accompanied by THHG in at least the latter two, which resolved spontaneously by the second trimester. In this patient, symptoms of nausea and vomiting as well as thyroid function normalized by 16.5 weeks gestation [43].

3.3. Multiple Pregnancies. Grün et al. [44] described hyperthyroidism in women with twin pregnancy, due to a much higher and more sustained peak of hCG. They collected 30 euthyroid women, in whom conception had been assisted by in vitro fertilization techniques, which allowed for the precise determination of gestational age, with 17 single pregnancies and 13 twin pregnancies. The peak hCG concentration between 8-11 weeks was significantly higher and much more prolonged in twin compared to single pregnancy (mean \pm SE $171,000 \pm 12,500$ versus $65,500 \pm 7600 \mathrm{U} / \mathrm{L} ; P<$ $0.001)$. Serum TSH values were lower and in 4 women serum FT4 were in the hyperthyroid range. None of the women complained of nausea or vomiting. Higuchi et al. [45] reported a triplet pregnancy, post-IVF treatment, developing hyperemesis at 6 weeks gestation, losing 14\% of her original body weight at 9 weeks gestation. Hyperthyroidism was diagnosed at 12 weeks' gestation, without evidence of autoimmunity. hCG titers were 359,900 mIU/L (expected between 12-20 weeks, less than 192,766 mIU/L). Vomiting, palpitations and headache did not resolve and she was treated with daily oral Lugol's solution from 13 weeks gestation, with alleviation of symptoms and improvement of thyroid tests; it was discontinued at 19 week's gestation. Thyroid tests were within normal limits in the triplets at birth and at one year of life. 
3.4. Hyperplacentosis. It is a condition of heightened trophoblastic activity characterized by increased placental weight and circulating hCG levels that are higher than those associated with normal pregnancy [46]. It may occur in association with diabetes, erythroblastosis, multiple pregnancies, and thalassemia. Clinically, it is characterized by tachycardia, heat intolerance, nausea, vomiting, and pruritus. The authors reported the first case in the literature of an association of hyperplacentosis and hyperthyroidism. When seen at 17 week's gestation, the patient had some nausea and vomiting but had gained weight normally throughout pregnancy. There were no signs of Graves' disease. Serum FT4 and FT3 were elevated and serum TSH was less than $0.3 \mathrm{mIU} / \mathrm{L}$. Pregnancy was terminated one week later because of a nonviable fetus. The placenta weight was $219 \mathrm{~g}$ (normal for gestational age 140-150 g) with histological findings consistent with Hyperplacentosis. The patient received medical therapy for a few days before the termination of pregnancy, with worsening of hypermetabolic symptoms, suggestive of thyroid storm, a day before surgical procedure. Thyroid tests returned to normal within several days and $\beta$-hCG normalized in within two months.

3.5. Hyperreactio Luteinalis. A rare condition characterized by pregnancy-associated ovarian enlargement due to the presence of multiple theca-lutein cysts discovered incidentally at the time of ultrasound, cesarean section, or postoperative tubal ligation, with the vast majority of cases asymptomatic. Gherman et al. [47] reported a case of a woman with intractable hyperemesis gravidarum, when seen at 27 weeks' gestation; she had thyroid tests consistent with hyperthyroidism and negative markers of thyroid autoimmunity. Because of severe vomiting, a $15 \mathrm{~kg}$ weight loss and dehydration, total peripheral nutrition was started, but discontinued one week later because of hypotension due to sepsis. Thyroid and liver tests normalized 9 days after hyperalimentation was started. An additional finding was virilization with high androgens levels diagnosed after delivery.

\footnotetext{
3.6. Familial Gestational Hyperthyroidism Caused by a Mutant Thyrotropin Receptor Hypersensitive to Human Chorionic Gonadotropin. In 1998 [48], a case was reported of a woman and her mother with an inheritable mutation of the thyroid TSH receptor, enhancing the thyrotropic influence of hCG. As a result, while the women had normal hCG levels during pregnancy, she developed severe nausea and vomiting, weight loss, persistent tachycardia, sweating, and hands tremor. Serum FT4 was elevated and serum TSH was undetectable. No antibodies (TPO or TRAb) were detected in serum. She required PTU therapy until 38 weeks gestation. A second pregnancy resulted in similar symptoms and course of treatment. The mother had had a similar medical history in her only full-term pregnancy. Both mother and daughter were heterozygous for a missense mutation, the K183R, in the extracellular domain of the thyrotropin receptor, highly sensitive to hCG, explaining the clinical picture in both mother and daughter.
}

3.7. Trophoblastic Diseases: Hydatidiform Mole and Choriocarcinoma. Hyperthyroidism has been reported in patients with trophoblastic tumors, either mole or choriocarcinoma. The incidence of hydatidiform mole has been reported to be between 0.5 and 2.5 per one thousand pregnancies [49]. The clinical presentation is characterized as vaginal bleeding suggesting threatened abortion. No fetal tissue is present, but the uterus is enlarged, larger than gestational age. Hyperemesis has been described in $20 \%$ of women with a high incidence of preeclampsia or eclampsia, before the time of ultrasonography as a diagnostic tool. The diagnosis is made by ultrasonography, demonstrating a "snowstorm" appearance without a fetus. With the use of routine sonography early in pregnancy for gestational age confirmation, and earlier pregnancy diagnosis, many of the reported complications are avoided. In a series comparing mole diagnosis in the period between 1965 to 1975 and 1994 to 1997 , evacuation of the mole was at 17.0 weeks versus 8.5 weeks, respectively, [50].

In 1955, Tisne et al. [51] reported the first cases of clinical and chemical hyperthyroidism in pregnant women with hydatidiform mole. The authors showed elevated radioactive iodine thyroid uptake in women with molar pregnancy as compared to normal pregnancy; one of their patients with a mole pregnancy had hyperthyroid symptoms. Soon thereafter, several investigators reported laboratory evidence of hyperthyroidism in women with molar pregnancy, with normalization of thyroid tests and reversal of hyperthyroid symptoms following evacuation of the mole. In 1963, Odell et al. [52] described a group of 93 patients with choriocarcinoma, seven of them with thyroid tests in the hyperthyroid range, but interestingly, none of them have symptoms or signs of hyperthyroidism. Thyroid tests returned to normal after evacuation of the mole. The authors found in the plasma of two of their patient's elevated thyrotropic activity by bioassay as well as in the extract of the tumor.

Hershman and Higgins [53] reported two cases of severe hyperthyroidism, one of them complicated by congestive heart failure, in patients with hydatidiform mole, and demonstrated for the first time thyrotropic activity in the molar tissue. This stimulator differed biologically and immunologically from the other three human stimulators: pituitary thyrotropin, chorionic thyrotropin found in normal pregnancies, and the long-acting thyroid stimulator (LATS) described by McKenzie in Graves' disease. The authors speculated that an excessive amount of the extracted molar stimulator was responsible for the hyperthyroidism in both patients that resolved following surgical extirpation of the moles. Galton et al. [54] reported the same year thyroid tests done in 11 patients, before and following the extraction of a molar pregnancy. They confirmed previous studies by Dowling et al. [55] that called attention of the striking abnormalities in several aspects of thyroid hormone economy in three patients with molar pregnancy. Consistent elevations in serum PBI, 24 hours 131 Iodine thyroid uptake, and serum total and free thyroxine were seen in the patients as compared to normal pregnant control patients. The prevalence of thyrotoxicosis in patients with trophoblastic tumors was reported to be close to $50 \%$ in some studies; 
TABLE 2: Hyperthyroidism and pregnancy clinical clues in the differential diagnosis.

\begin{tabular}{lcc}
\hline & Gestational & Graves' \\
\hline Symptoms prepregnancy & - & ++ \\
Symptoms during pregnancy & $-/+$ & $+/+++$ \\
Nausea/vomiting & ++++ & $-/+$ \\
Goiter/ophthalmopathy & - & + \\
Anti-TPO/TSHRAb & - & + \\
TT3/TT4 ratio & $<20$ & $>20$ \\
\hline
\end{tabular}

nowadays, with the ability of early detection of the disease, the incidence is much lower although still reported.

The diagnosis of trophoblastic disease is confirmed by ultrasonography and the presence of high levels of the $\beta$ subunit of hCG [3] that serves as a marker for the tumor. Therapy of hyperthyroidism is not indicated in the vast majority of cases, since evacuation of the mole or chemotherapy for management of choriocarcinoma removing high levels of hCG cures the hyperthyroidism. In those cases of severe symptoms, Lugol's solution, IV iodine, $\beta$-blocking agents and sometimes TD are indicated. A case of thyroid storm was reported [56].

3.8. Hyperemesis Gravidarum in Women with Graves' Disease. Women diagnosed with Graves' hyperthyroidism before pregnancy and in remission may have a flare up of the symptoms during the first trimester, secondary to the hCG and TRAb stimulating effect on the thyroid TSH receptor. The situation may be further complicated by the presence of severe vomiting in women with a previous diagnosis of Graves' hyperthyroidism. Tagami et al. [57] studied 39 pregnancies in 334 women with Graves' disease. Ten of the women $(26 \%)$ had an episode of gestational hyperthyroidism; thyroid function tests, antibodies determination and measurement of hCG are of relative value in the differential diagnosis, because there is significant overlap between the two groups. A high T3/T4 ratio could be of diagnostic value, favoring Graves' hyperthyroidism when the ratio is $>20$.

\section{Clinical and Laboratory Diagnosis}

One of the most challenging situations in the presence of hyperthyroid thyroid tests early in pregnancy, is in the differential diagnosis between hCG induced hyperthyroidism and Graves' hyperthyroidism. Since the first recognition of the association between HG and hyperthyroidism, several small series (less than 100 patients each) have been reported. The most important tools in the evaluation of women with hyperthyroidism in the first trimester of pregnancy are a careful history and detailed and targeted physical examination. From all publications reviewed, a clear picture had developed both from the physiopathological and clinical point of view. These can be summarized as follows:
(1) a previous healthy woman presents by the 4-9th weeks' gestation with hyperemesis gravidarum, manifested by severe nausea, vomiting, and weight loss.

(2) depending on the severity of dehydration, hospitalization will be required in a significant number of patients.

(3) in up to $70 \%$ of patients, laboratory tests are consistent with hyperthyroidism, diagnosed by a suppressed or undetectable serum TSH with an elevation of serum FT4. Serum TT3 is rarely necessary to confirm the diagnosis. Very few patients have clinical hyperthyroid manifestations. Serum HCG concentrations are not helpful as a diagnostic tool, with few exceptions (hydatidiform mole and choriocarcinoma).

(4) the following features define the diagnosis in most patients and assist the physicians in the differential diagnosis from Graves' hyperthyroidism (Table 2):

(a) medical history:

(i) absence of hyperthyroid symptoms before conception,

(ii) similar history of vomiting in previous pregnancies,

(iii) family history of Hyperemesis Gravidarum,

(iv) no previous history of thyroid disease,

(b) physical examination:

(i) no goiter,

(ii) no Graves' ophthalmopathy,

(iii) no other physical findings such as vitiligo and Plummer's nails,

(iv) signs of dehydration,

(c) laboratory tests:

(i) FT4 or FT4I elevated,

(ii) TSH suppressed or undetectable,

(iii) negative thyroid antibodies: TPO and TRAb,

(iv) determination of TT3 or FT3 indicated when strong suspicion of Graves' hyperthyroidism or presence of a dominant thyroid nodule,

(v) the incidence of reported transient electrolytes abnormalities is about $60 \%$ and liver abnormalities about $50 \%$ of patients,

(vi) abdominal ultrasound to rule out multiple pregnancies or the presence of a hydatidiform mole,

(vii) thyroid ultrasonography: data not available in the literature,

(5) in the vast majority of situations, vomiting subsidies after 14-18 weeks gestation,

(6) serum FT4 returned to normal by the 15th week gestation or before, 
(7) serum TSH may remained suppressed well into the end of the second trimester,

(8) obstetrical complications do not appear to be significant. The only consistent findings reported in several publications is a lower birth weight as compared to infants born to mothers not afflicted by the disease and same gestational age. Low birth weight is related to severity of vomiting and degree of weight loss and is not correlated to the transient maternal hyperthyroidism [58].

\section{Management}

Hyperemesis gravidarum management includes intravenous hydration, containing vitamin B complex, and nausea medications: for women with persistent vomiting, significant weight loss, and presence of ketones in urine, hospitalization is very frequently required. Parenteral nutrition or nasogastric feeding tube is required in a minority of affected women. One of the most devastating complications of HG, although extremely rare, is Wernicke encephalopathy [59]. ATD therapy is not recommended, and no prospective study is available comparing obstetrical outcome among women receiving treatment and control group. Very few patients from the published series were treated with ATD and showed no benefit from it. One of the clinical problems with ATD therapy is the poor tolerability by patients, because of the persistent vomiting and metal taste particularly of PTU. In one occasion, PTU suppository was employed; the hyperthyroidism resolved by 18 weeks but vomiting persisted and ceased at 27 weeks, 3 days after termination of pregnancy [60]. As mentioned above [45], one case of severe hyperthyroxinemia in a triplet pregnancy the symptoms improved with the combination of Lugol's solution and PTU.

\section{Summary}

Transient nonimmune hyperthyroidism of early pregnancy is defined as an episode of transient hyperthyroidism, without evidence of thyroid autoimmunity, lack of Graves' disease physical findings, resolving spontaneously by the end of the first or early second trimester of pregnancy. Of the several causes, hyperemesis gravidarum is the most common one. The challenge for the attending physician is the differential diagnosis from Graves' disease. High levels of hCG or a hCG molecule variant with high biological activity, stimulating the TSH receptor induces hyperthyroidism. Consistent laboratory findings are a suppressed of undetectable serum TSH, an elevation in serum FT4 and negative tests for autoimmunity (TPO and TRAb). Hyperthyroidism resolves with cessation of vomiting by 14-16 weeks gestation although serum TSH may remained suppressed for a few more weeks. Fetal ultrasound is indicated to rule out other causes of THHG, such as multiple pregnancies and trophoblastic disease. ATD therapy is rarely indicated; treatment is based on correction of hydration and electrolytes replacement. Obstetrical outcome is not affected, with the exception in some series of lower infant weight at birth.

\section{References}

[1] D. Glinoer, P. de Nayer, P. Bourdoux et al., "Regulation of maternal thyroid during pregnancy," Journal of Clinical Endocrinology and Metabolism, vol. 71, no. 2, pp. 276-287, 1990.

[2] G. E. Krassas, K. Poppe, and D. Glinoer, "Thyroid function and human reproductive health," Endocrine Reviews, vol. 31, no. 5, pp. 702-755, 2010.

[3] C. M. Lockwood, D. G. Grenache, and A. M. Gronowski, "Serum human chorionic gonadotropin concentrations greater than 400,000 IU/L are invariably associated with suppressed serum thyrotropin concentrations," Thyroid, vol. 19, no. 8, pp. 863-868, 2009.

[4] G. Glinoer and C. A. Spencer, "Serum TSH determinations in pregnancy: how, when and why?" Nature Reviews Endocrinology, vol. 6, pp. 526-529, 2010.

[5] T. M. Goodwin, M. Montoro, and J. H. Mestman, "Transient hyperthyroidism andhyperemesis gravidarum: clinical aspects," American Journal of Obstetrics and Gynecology, vol. 167, no. 3, pp. 648-652, 1992.

[6] T. M. Goodwin and J. M. Hershman, "Hyperthyroidism due to inappropriate production of Human Chorionic Gonadotropin," Clinical Obstetrics and Gynecology, vol. 40, no. 1, pp. 32-44, 1997.

[7] M. Kimura, N. Amino, H. Tamaki et al., "Gestational thyrotoxicosis and hyperemesis gravidarum: possible role of hCG with higher stimulating activity," Clinical Endocrinology, vol. 38, no. 4, pp. 343-350, 1993.

[8] M. Mori, N. Amino, H. Tamaki, K. Miyai, and O. Tanizawa, "Morning sickness and thyroid function in normal pregnancy," Obstetrics and Gynecology, vol. 72, no. 3, pp. 355-359, 1988.

[9] T. M. Goodwin, "Hyperemesis gravidarum," Clinical Obstetrics and Gynecology, vol. 41, no. 3, pp. 597-605, 1998.

[10] J. R. Niebyl, "Nausea and vomiting in pregnancy," The New England Journal of Medicine, vol. 363, no. 16, pp. 1544-1550, 2010.

[11] M. M. Weigel and R. M. Weigel, "Nausea and vomiting of early pregnancy and pregnancy outcome. An epidemiological study," British Journal of Obstetrics and Gynaecology, vol. 96, no. 11, pp. 1304-1311, 1989.

[12] K. Patil-Sisodia and J. H. Mestman, "Graves hyperthyroidism and pregnancy: a clinical update," Endocrine Practice, vol. 16, no. 1, pp. 118-129, 2010.

[13] T. M. Goodwin, M. Montoro, and J. H. Mestman, "The role of chorionic gonadotropin in transient hyperthyroidism of hyperemesis gravidarum," Journal of Clinical Endocrinology and Metabolism, vol. 75, no. 5, pp. 1333-1337, 1992.

[14] A. M. Grjibovski, Å. Vikanes, C. Stoltenberg et al., "Consanguinity and the risk of hyperemesis gravidarum in Norway," Acta Obstetricia et Gynecologica Scandinavica, vol. 12, pp. 1-6, 2007.

[15] J. L. Bailit, "Hyperemesis gravidarium: epidemiologic findings from a large cohort," American Journal of Obstetrics and Gynecology, vol. 193, no. 3, pp. 811-814, 2005.

[16] Y. Zhang, R. M. Cantor, K. MacGibbon et al., "Familial aggregation of hyperemesis gravidarum," American Journal of Obstetrics and Gynecology, vol. 204, no. 3, pp. 230.e1-230.e7, 2011.

[17] M. S. Fejzo, R. Romero, and T. M. Goodwin, "Patients with a history of hyperemesis gravidarum have similar symptoms during egg stimulation and develop ovarian hyperstimulation syndrome: case series," Fertility and Sterility, vol. 93, no. 1, pp. 267.e9-267.e11, 2010. 
[18] J. M. Hershman, "Physiological and pathological aspects of the effect of human chorionic gonadotropin on the thyroid," Best Practice and Research: Clinical Endocrinology and Metabolism, vol. 18, no. 2, pp. 249-265, 2004.

[19] M. Yoshimura, J. H. Hershman, X. P. Pang et al., "Activation of the thyrotropin (TSH) receptor by human chorionic gonadotropin and luteinizing hormone in Chines hamster ovary cell expressing functional human TSH receptors," The Journal of Clinical Endocrinology and Metabolism, vol. 77, pp. 1009-1013, 1993.

[20] J. M. Hershman, "Editorial: role of human chorionic gonadotropin as a thyroid stimulator," Journal of Clinical Endocrinology and Metabolism, vol. 74, no. 2, pp. 258-259, 1992.

[21] A. Kauppila, I. Huhtaniemi, and O. Ylikorkala, "Raised serum human chorionic gonadotrophin concentrations in hyperemesis gravidarum," British Medical Journal, vol. 1, no. 6179, pp. 1670-1671, 1979.

[22] R. L. Kennedy, R. Davies, and A. Price, "Thyrotoxicosis and hyperemesis gravidarum associated with a serum activity which stimulates human thyroid cells in vitro," Clinical Endocrinology, vol. 36, no. 1, pp. 63-89, 1992.

[23] A. E. Pekary, I. M. D. Jackson, T. M. Goodwin, X. P. Pang, M. D. Hein, and J. M. Hershman, "Increased in vitro thyrotropic activity of partially sialated human chorionic gonadotropin extracted from hydatidiform moles of patients with hyperthyroidism," Journal of Clinical Endocrinology and Metabolism, vol. 76, no. 1, pp. 70-74, 1993.

[24] E. Tsuruta, H. Tada, H. Tamaki et al., "Pathogenic role of asialo human chorionic gonadotropin in gestational thyrotoxicosis," Journal of Clinical Endocrinology and Metabolism, vol. 80, no. 2, pp. 350-355, 1995.

[25] V. Jordan, S. K. G. Grebe, R. R. Cooke et al., "Acidic isoforms of chorionic gonadotrophin in European and Samoan women are associated with hyperemesis gravidarum and may be thyrotrophic," Clinical Endocrinology, vol. 50, no. 5, pp. 619$627,1999$.

[26] T. Bruun and K. Kristofferson, "Thyroid function during pregnancy with special reference to hydatidiform mole and hyperemesis," Acta Endocrinologica, vol. 88, no. 2, pp. 383-389, 1978.

[27] B. H. Valentine and C. Jones, "Hyperemesis gravidarum due to thyrotoxicosis," Postgraduate Medical Journal, vol. 56, no. 660, pp. 746-747, 1980.

[28] F. D. Rosenthal, C. Jones, and S. I. Lewias, "Thyrotoxic vomiting," British Medical Journal, vol. 2, no. 6029, pp. 209-211, 1976.

[29] R. Dozeman, F. L. Kaiser, O. Cass, and J. Pries, "Hyperthyroidism appearing as hyperemesis gravidarum," Archives of Internal Medicine, vol. 143, no. 11, pp. 2202-2203, 1983.

[30] W. J. Jeffcoate and C. Bain, "Recurrent pregnancy-induced thyrotoxicosis presenting as hyperemesis gravidarum. Case report," British Journal of Obstetrics and Gynaecology, vol. 92, no. 4, pp. 413-415, 1985 .

[31] R. Bouillon, M. Naesens, F. A. Van Assche et al., "Thyroid function in patients with hyperemesis gravidarum," American Journal of Obstetrics and Gynecology, vol. 143, no. 8, pp. 922926, 1982.

[32] N. Juras, K. Banovac, and M. Sekso, "Increased serum reverse triiodothyronine in patients with hyperemesis gravidarum," Acta Endocrinologica, vol. 102, no. 2, pp. 284-287, 1983.

[33] H. Asakura, S. Watanabe, A. Sekiguchi, G. G. Power, and T. Araki, "Severity of hyperemesis gravidarum correlates with serum levels of reverse T3," Archives of Gynecology and Obstetrics, vol. 264, no. 2, pp. 57-62, 2000.

[34] M. Montoro, C. Spencer, S. Jacobson et al., "Evidence for a physiological role of hCG as a thyroid stimulator: genesis of hyperthyroidism in hyperemesis gravidarum," Clinical Research, vol. 32, p. 20A, 1984.

[35] S. A. Bober, A. C. McGill, and W. M. G. Tunbridge, "Thyroid function in hyperemesis gravidarum," Acta Endocrinologica, vol. 111, no. 3, pp. 404-410, 1986.

[36] T. T. Lao, R. K. H. Chin, and A. M. Z. Chang, "The outcome of hyperemetic pregnancies complicated by transient hyperthyroidism," Australian and New Zealand Journal of Obstetrics and Gynaecology, vol. 27, no. 2, pp. 99-101, 1987.

[37] A. Price, R. Davies, S. R. Heller, A. Milford-Ward, and A. P. Weetman, "Asian women are at increased risk of gestational thyrotoxicosis," Journal of Clinical Endocrinology and Metabolism, vol. 81, no. 3, pp. 1160-1163, 1996.

[38] C. P. Yeo, D. H. C. Khoo, P. H. K. Eng, H. K. Tan, S. L. Yo, and E. Jacob, "Prevalence of gestational thyrotoxicosis in Asian women evaluated in the 8th to 14 th weeks of pregnancy: correlations with total and free beta human chorionic gonadotrophin," Clinical Endocrinology, vol. 55, no. 3, pp. 391-398, 2001.

[39] T. J. Caffrey, "Transient hyperthyroidism of hyperemesis gravidarum: a sheep in wolf's clothing," Journal of the American Board of Family Practice, vol. 13, no. 1, pp. 35-38, 2000.

[40] P. Deruelle, P. Dufour, D. Subtil et al., "Hyperemesis in the first trimester of pregnancy: role of biological hyperthyroidism and fetal sex," Gynecologie Obstetrique Fertilite, vol. 30, no. 3, pp. 204-209, 2002.

[41] N. S. Panesar, C. Y. Li, and M. S. Rogers, "Are thyroid hormones or hCG responsible for hyperemesis gravidarum? A matched paired study in pregnant Chinese women," Acta Obstetricia et Gynecologica Scandinavica, vol. 80, no. 6, pp. 519-524, 2001.

[42] J. Y. L. Tan, K. C. Loh, G. S. H. Yeo, and Y. C. Chee, “Transient hyperthyroidism of hyperemesis gravidarum," An International Journal of Obstetrics and Gynaecology, vol. 109, no. 6, pp. 683-688, 2002.

[43] S. Nader and J. Mastrobattista, "Recurrent hyperthyroidism in consecutive pregnancies characterized by hyperemesis," Thyroid, vol. 6, no. 5, pp. 465-466, 1996.

[44] J. P. Grün, S. Meuris, P. De Nayer, and D. Glinoer, "The thyrotrophic role of human chorionic gonadotrophin (hCG) in the early stages of twin (versus single) pregnancies," Clinical Endocrinology, vol. 46, no. 6, pp. 719-725, 1997.

[45] R. Higuchi, S. Minami, S. Yagi et al., "Gestational thyrotoxicosis during a triplet pregnancy," Journal of Obstetrics and Gynaecology, vol. 28, no. 4, pp. 444-445, 2008.

[46] J. Ginsberg, R. Z. Lewanczuk, and L. H. Honore, "Hyperplacentosis: a novel cause of hyperthyroidism," Thyroid, vol. 11, no. 4, pp. 393-396, 2001.

[47] R. B. Gherman, J. H. Mestman, A. J. Satin, and T. M. Goodwin, "Intractable hyperemesis gravidarum, transient hyperthyroidism and intrauterine growth restriction associated with hyperreactio luteinalis: a case report," Journal of Reproductive Medicine for the Obstetrician and Gynecologist, vol. 48, no. 7, pp. 553-556, 2003.

[48] P. Rodien, C. Brémont, M. L. R. Sanson et al., "Familial gestational hyperthyroidism caused by a mutant thyrotropin receptor hypersensitive to human chorionic gonadotropin," The New England Journal of Medicine, vol. 339, no. 25, pp. 18231826, 1998. 
[49] R. K. Desai, R. J. Norman, I. Jialal, and S. M. Joubert, "Spectrum of thyroid function abnormalities in gestational trophoblastic Neoplasia," Clinical Endocrinology, vol. 29, no. 6, pp. 583-592, 1988.

[50] R. Mosher, D. P. Goldstein, R. Berkowitz et al., "Complete hydatidiform mole: comparison of clinicopathologic features, current and past," Journal of Reproductive Medicine for the Obstetrician and Gynecologist, vol. 44, pp. 698-704, 1999.

[51] L. Tisne, J. Barzelatto, and C. Stevenson, "Study of thyroid function during pregnancy and postpartum period with radioactive iodine (Spanish)," Bol de la Sociedad Chilena de Obstetrica y Ginecologia, vol. 20, p. 246, 1955.

[52] W. D. Odell, R. W. Bates, R. S. Rivlin, M. B. Lipsett, and R. Hertz, "Increased thyroid function without clinical hyperthyroidism in patients with choriocarcinoma," The Journal of Clinical Endocrinology and Metabolism, vol. 23, pp. 658-664, 1963.

[53] J. M. Hershman and H. P. Higgins, "Hydatidiform mole-a cause of clinical hyperthyroidism. Report of two cases with evidence that the molar tissue secreted a thyroid stimulator," The New England Journal of Medicine, vol. 284, no. 11, pp. 573577, 1971.

[54] V. A. Galton, S. H. Inggar, J. Jimenez-Fonseca, and J. M. Hershman, "Alterations in thyroid hormone economy in patients with hydatidiform mole," Journal of Clinical Investigation, vol. 50, no. 6, pp. 1345-1354, 1971.

[55] J. T. Dowling, J. H. Ingbar, and N. Freinkel, "Iodine metabolism in hydatidiform mole and choriocarcinoma," The Journal of Clinical Endocrinology and Metabolism, vol. 20, pp. 1-12, 1960.

[56] N. U. Chiniwala, P. D. Wool, C. P. Bruno, S. Kaur, H. Spector, and K. Yacono, "Thyroid storm caused by a partial hydatidiform mole," Thyroid, vol. 18, no. 4, pp. 479-481, 2007.

[57] T. Tagami, H. Hagiwara, T. Kimura, T. Usui, A. Shimatsu, and M. Naruse, "The incidence of gestational hyperthyroidism and postpartum thyroiditis in treated patients with Graves' disease," Thyroid, vol. 17, no. 8, pp. 787-772, 2007.

[58] S. Gross, C. Librach, and A. Cecutti, "Maternal weight loss associated with hyperemesis gravidarum: a predictor of fetal outcome," American Journal of Obstetrics and Gynecology, vol. 160, no. 4, pp. 906-909, 1989.

[59] N. Ohmori, T. Tushima, Y. Sekine et al., "Gestational thyrotoxicosis withacute Wernicke encephalopathy: a case report," Endocrine Journal, vol. 46, no. 6, pp. 787-793, 1999.

[60] B. Kirshon, W. Lee, and D. B. Cotton, "Prompt resolution of hyperthyroidism and hyperemesis gravidarum after delivery," Obstetrics and Gynecology, vol. 71, no. 6, pp. 1032-1034, 1988. 


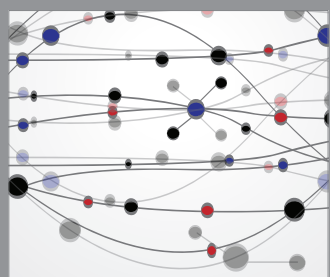

The Scientific World Journal
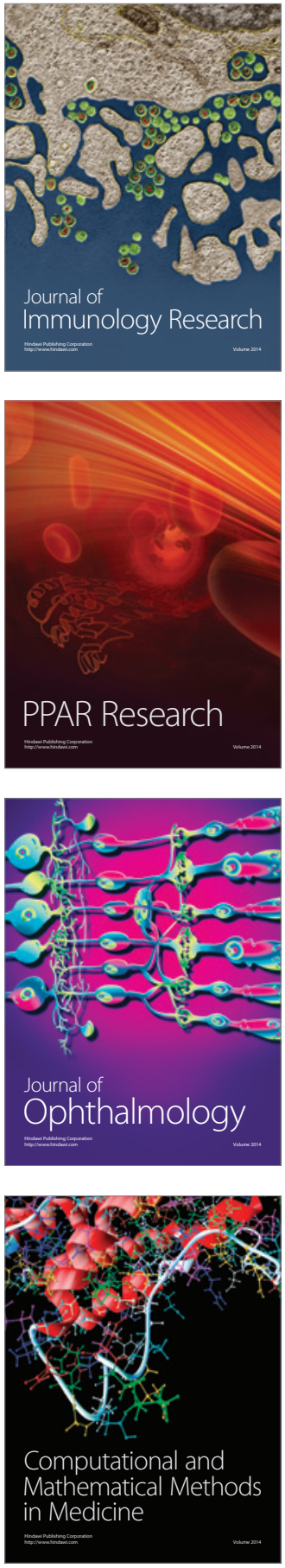

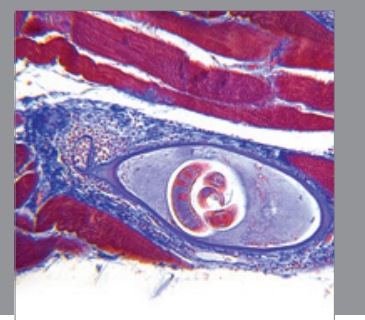

Gastroenterology

Research and Practice
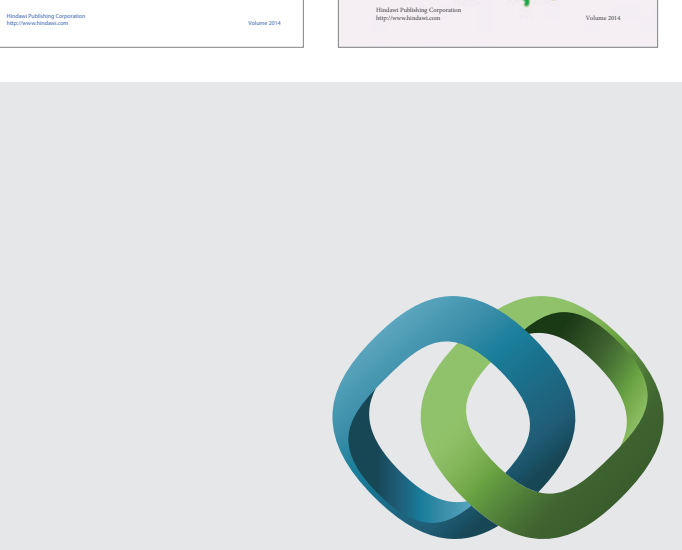

\section{Hindawi}

Submit your manuscripts at

http://www.hindawi.com
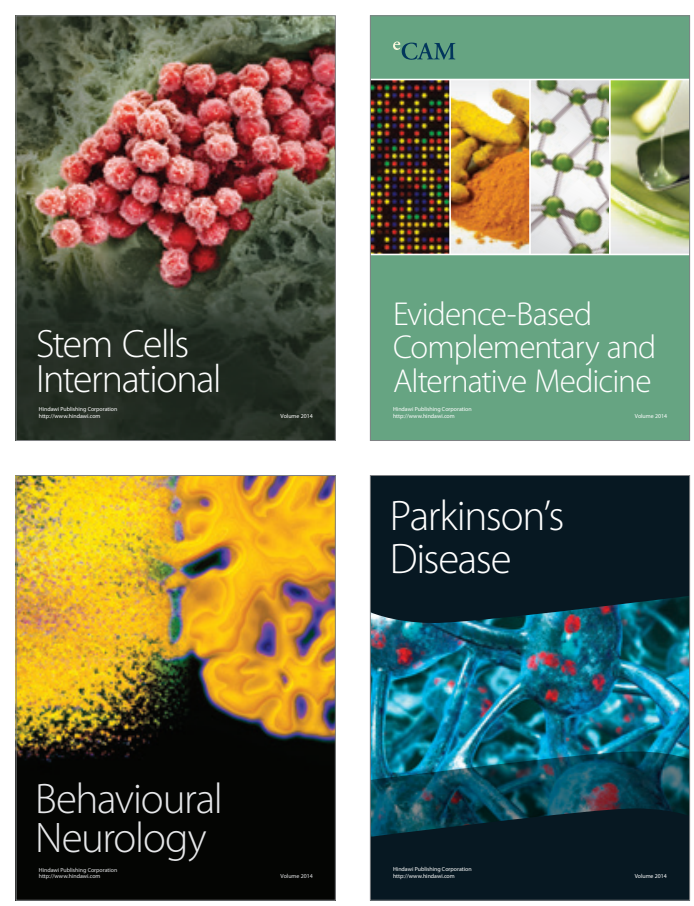

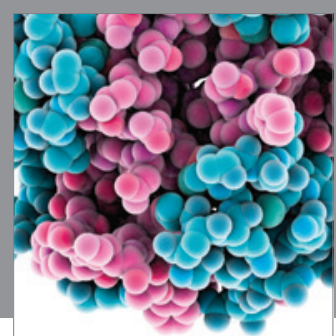

Journal of
Diabetes Research

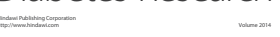

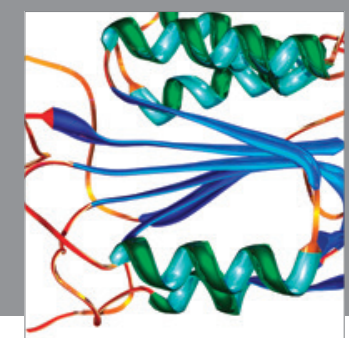

Disease Markers
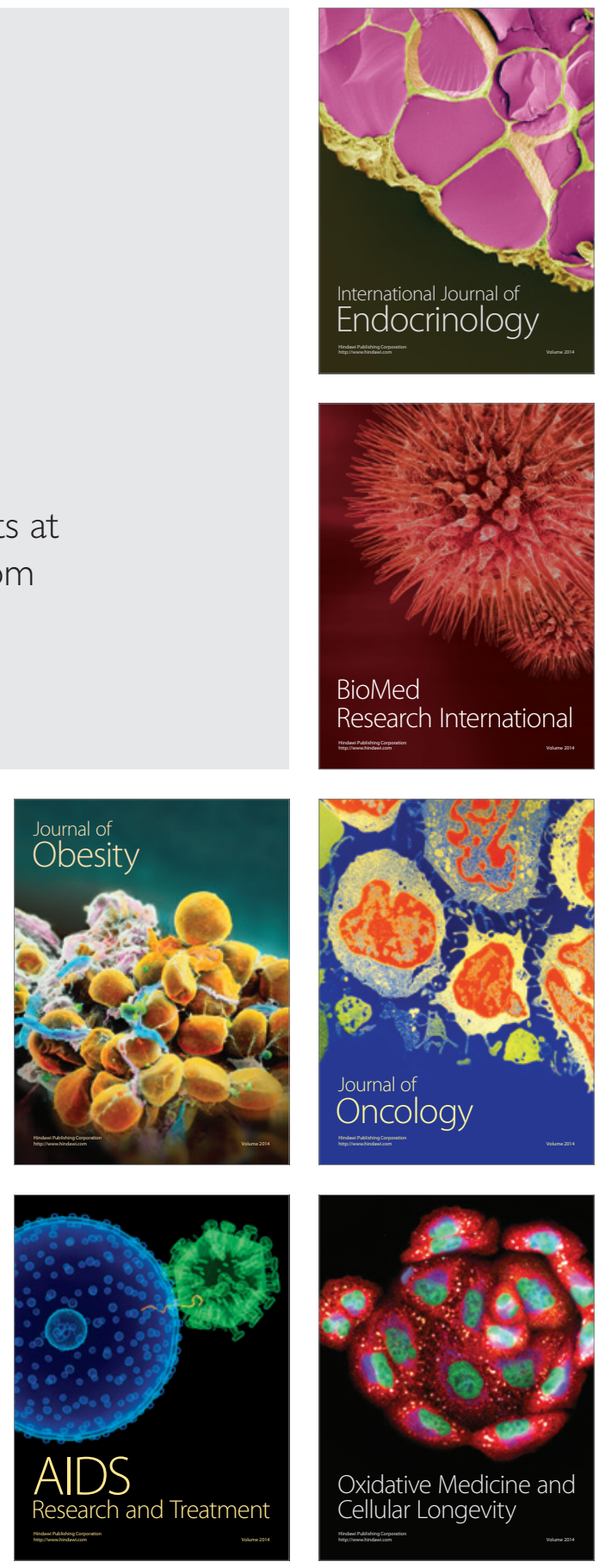